\title{
Quarkonium production review
}

\section{Kuang-Ta Chao*}

Department of Physics and State Key Laboratory of Nuclear Physics and Technology, and Center for High Energy Physics, Peking University, Beijing 100871, China

E-mail: ktchaodpku.edu.cn

\section{Yan-Qing Ma}

Physics Department, Brookhaven National Laboratory, Upton, NY 11973, USA

E-mail: vamadbn1.gov

Recent progress in heavy quarkonium production is reviewed in the framework of nonrelativistic QCD. Possible resolutions of the long standing problems of $J / \psi$ and $\psi^{\prime}$ production cross sections and polarizations at high $p_{T}$ observed at the Tevatron are investigated, and predictions are compared with the new measurements at the LHC. Puzzles in hadroproduction of P-wave charmonium states $\chi_{c J}$ are also discussed. In addition, charmonium production in $e^{+} e^{-}$annihilation at $\mathrm{B}$ factories and in photoproduction at HERA as well as in two-photon collisions are also reviewed. Special emphasis is put on the role of next-to-leading order corrections in NRQCD, which may essentially change the $p_{T}$ distributions at high $p_{T}$ for both yield and polarization in quarkonium hadroproduction.

Xth Quark Confinement and the Hadron Spectrum,

October 8-12, 2012

TUM Campus Garching, Munich, Germany

\footnotetext{
* Speaker.
} 


\section{Introduction}

The study of heavy quarkonium production is expected to provide important information for perturbative and nonperturbative QCD. Because heavy quark masses $\left(m_{c} \approx 1.3 \mathrm{GeV}\right.$ and $m_{b} \approx$ $4.2 \mathrm{GeV}$ in $\overline{\mathrm{MS}}$ scheme) are much larger than $\Lambda_{Q C D}$, the typical scale where nonperturbative effects become significant, it is believed that one can use factorization method to describe heavy quarkonium production. The currently widely used theoretical framework is Non-Relativistic QCD (NRQCD) factorization[四]. Thanks to the break though of higher order calculations in QCD, theoretical study for quarkonium production has made much progress in recent years (for a comprehensive review of quarkonium physics including production, see Ref.[వ]]).

In this talk, we review the current status of the theoretical framework for inclusive quarkonium production and the comparison between theoretical predictions and experimental measurements. We put stress on the issues of charmonium and bottomonium production at hadron colliders such as the Tevatron and the LHC, and also in $e^{+} e^{-}$annihilation at B factories as well as in $e p$ and $\gamma \gamma$ collisions.

\section{Factorization methods for quarkonium production}

Since the discovery of $J / \psi$ in 1974 , a number of factorization approaches in QCD have been proposed to describe quarkonium production: from the color-singlet model (CSM) [3], 田, []] and color-evaporation model (CEM) [ $\mathbf{6}, \square, \mathbf{\square}, \mathbf{Q}]$, to a more general method, NRQCD factorization, proposed by Bodwin, Braaten and Lepage in 1994[四]. More recently, the fragmentation approach was also proposed [ए, ए] to deal with quarkonium production at large transverse momentum $p_{T}$ in $1 / p_{T}$ expansion.

For inclusive quarkonium $H$ production, the NRQCD factorization form is [四]:

$$
\sigma(H)=\sum_{n} F_{n}\left\langle\mathscr{O}_{n}^{H}\right\rangle,
$$

where $n$ denote quantum numbers for the intermediate states which hadronize to quarkonium $H$ ultimately. The long-distance matrix element (LDME) $\left\langle\mathscr{O}_{n}^{H}\right\rangle$, which has a known scaling with $v$ (the heavy-quark velocity in quarkonium rest frame), describes the probability of the hadronization. The values of LDMEs may be determined by fitting some experimental data. It is important to note that, if NRQCD factorization works, the LDMEs should be universal (independent of process). $F_{n}$ are the short-distance coefficients and they depend only on large energy scales such as heavy quark mass $m$ and transverse momentum $p_{T} . F_{n}$ are process dependent, and they can be calculated in perturbative QCD by expansion in powers of $\alpha_{s}$, based on the matching relation in Eq.(2. $(1)$ ). Hence, by using NRQCD factorization, one can do a double expansion in powers of $\alpha_{s}$ and $v$. In practice, within a certain precision, one can truncate the expansion to a finite order in $v$. For example, only the following LDMEs are frequently studied in $1^{--}$quarkonium $\left(J / \psi, \psi^{\prime}\right.$ and $\left.\Upsilon(n S)\right)$ production:

$$
\left\langle\mathscr{O}^{H}\left({ }^{3} S_{1}^{[1]}\right)\right\rangle, \quad\left\langle\mathscr{O}^{H}\left({ }^{1} S_{0}^{[8]}\right)\right\rangle, \quad\left\langle\mathscr{O}^{H}\left({ }^{3} S_{1}^{[8]}\right)\right\rangle, \quad\left\langle\mathscr{O}^{H}\left({ }^{3} P_{J}^{[8]}\right)\right\rangle,
$$

which are relatively suppressed by $v^{0}, v^{3}, v^{4}$ and $v^{4}$, respectively [四].

As shown above, in NRQCD factorization there are both color-singlet (CS) channel and coloroctet (CO) channel contributions. If one set color-octet contributions to be zero, one would recover 
the CSM. It is also shown that CEM can be only a special case of NRQCD factorization by choosing specific LDMEs[[12] $]^{1}$. Although NRQCD factorization is general enough to include CSM and CEM, the CSM is still attractive because it is a necessary part of NRQCD, and it basically contains no unknown parameters (the LDMEs for CSM can be reasonably estimated, e.g., using potential model).

Unfortunately, to prove NRQCD factorization is hard. Nayak, Qiu, and Sterman find that, in order to make the CO LDMEs gauge invariant, one must modify them from the original definition by including eikonal lines which run from the hard point to infinity [[13], [4]]. After adding these eikonal lines, factorization is valid up to two-loop order [[13], [4]]. But an all-order proof is still missing ${ }^{2}$.

The only factorization theory for quarkonium production that has been proven at present is

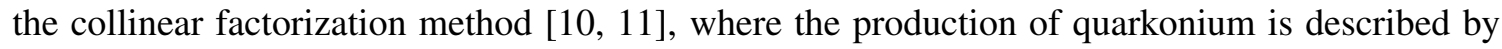
one particle fragmentation function (LP) and two particle fragmentation (NLP). Generally, these fragmentation functions should be determined by fitting data. However, if NRQCD factorization is valid, one can apply NRQCD factorization to calculate these fragmentation functions (e.g. [ㅍ], [8, [1], [9]). In this case, the collinear factorization can be used to reorganize the power expansion of $1 / p_{T}$ and to resum large $\log$ arithms $\log \left(p_{T}^{2} / \mathrm{m}^{2}\right)$ in the calculation using NRQCD factorization. Anyway, this collinear factorization should be very helpful to understand quarkonium production, but still lots of work need to be done.

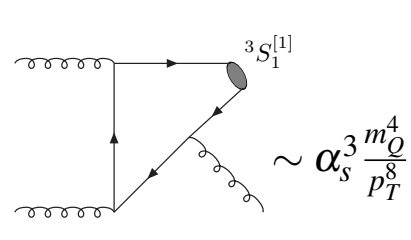

(a)

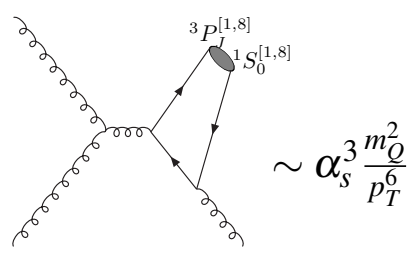

(d)

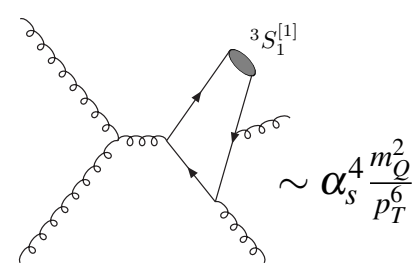

(b)

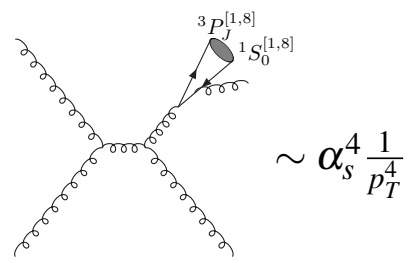

(e)

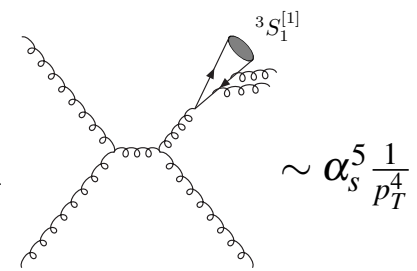

(c)

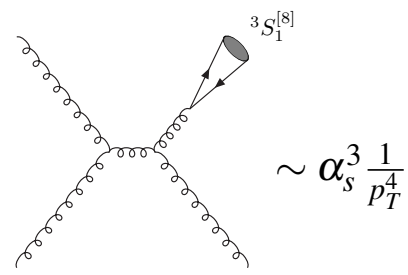

(f)

Figure 1: Typical Feynman diagrams for quarkonium hadroproduction in NRQCD. $p_{T}$ behavior of partonic differential cross section $\frac{d \sigma}{d p_{T}^{2}}$ for each diagram is also given.

\footnotetext{
${ }^{1}$ In NRQCD factorization, one do expansion in powers of $v$, while in CEM finite $v$ effects are included. Thus the argument in Ref.[ए2] makes sense only if $v$ is small enough to validate the power expansion.

${ }^{2}$ Nayak, Qiu, and Sterman also find that the NRQCD factorization picture will be broken down if an additional heavy quark is also produced [ए5], [0]].
} 


\section{Quarkonium production at hadron colliders}

\subsection{History of LO predictions and polarization puzzle}

CSM can give good descriptions for earlier data of quarkonium production until the early 90's. In 1994, by subtracting contributions from $B$ decay, the prompt $\psi^{\prime}$ production rate was found to be unexpectedly large [[D] $]$. Even after including the gluon fragmentation contribution, the leading order (LO) CSM predictions for prompt $\psi^{\prime}$ production are still smaller than the data by a factor of 30 when $p_{T}$ is larger than $10 \mathrm{GeV}$ [2], 22], 23]]. This was called the $\psi^{\prime}$ surplus anomaly.

The $\psi^{\prime}$ anomaly can be well understood in NRQCD factorization. As indicated in Eq.([2.2), $\psi^{\prime}$ hadroproduction in NRQCD factorization has both CS channel ${ }^{3} S_{1}^{[1]}$ and CO channel ${ }^{3} S_{1}^{[8]},{ }^{[} S_{0}^{[8]}$, and ${ }^{3} P_{J}^{[8]}$ contributions up to $O\left(v^{4}\right)$. At LO in $\alpha_{s}$ and for high $p_{T}$ regions, it can be shown that the partonic differential cross section of ${ }^{3} S_{1}^{[1]}$ has $p_{T}^{-8}$ behavior (Fig. प(a)), ${ }^{1} S_{0}^{[8]}$ and ${ }^{3} P_{J}^{[8]}$ have $p_{T}^{-6}$ behavior (Fig. प(d)), while ${ }^{3} S_{1}^{[8]}$ has $p_{T}^{-4}$ behavior (Fig. प(f)) [24]. Therefore, at high $p_{T}$ the CO channels will be more important than CS. By including CO contributions, the LO NRQCD can give a good description for the yield of prompt $\psi^{\prime}$ production at high $p_{T}[25,[26]$.

Although it seemed successful to explain the differential cross sections for quarkonium production, NRQCD factorization encounters difficulties when charmonia polarizations are taken into consideration. Dominated by gluon fragmentation to ${ }^{3} S_{1}^{[8]}$ at large $p_{T}$, LO NRQCD predicts a sizable transverse polarization for both $J / \psi$ and $\psi^{\prime}$ [27, 228, 229], while the Tevatron measurement gives almost unpolarized results [30], see Fig. $\square$ for the newest measurement at the Tevatron compared with LO NRQCD prediction. This polarization puzzle became the main obstacle to understand the production mechanism of heavy quarkonium in the past decade [[]].
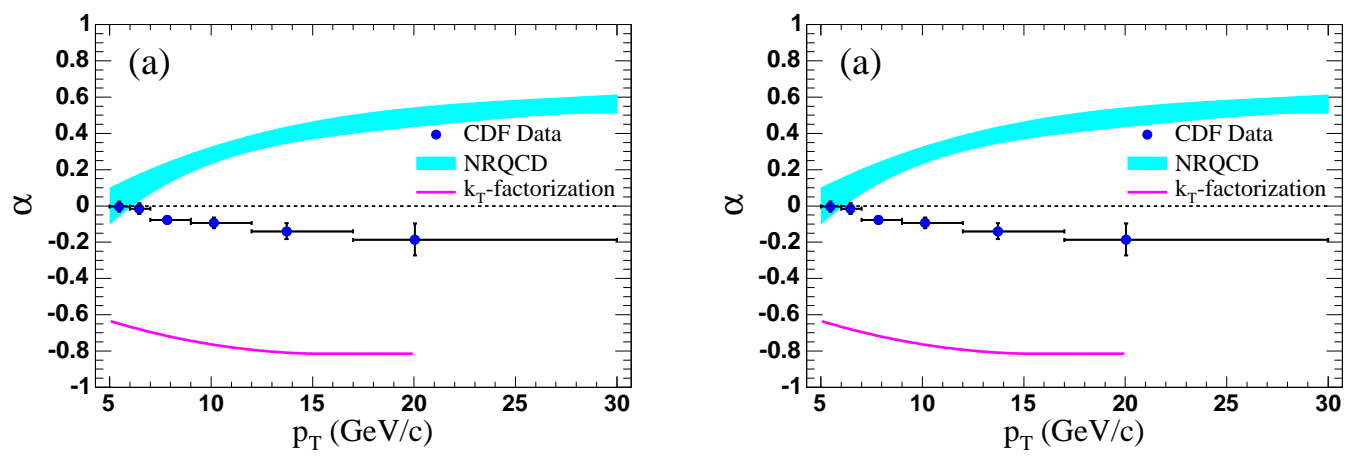

Figure 2: Prompt polarizations as functions of $p_{T}$ : (a) $J / \psi$ and (b) $\psi^{\prime}$. The band (line) is the prediction from NRQCD [27, 28, 22] (the $k_{T}$-factorization model [B]]). (Figure taken from Ref. [B2].)

When $p_{T}$ is very large, however, contributions at high order in $\alpha_{s}$ may become very important. As noted in Fig. 四, $d \sigma / d p_{T}^{2}$ behaviors for CS channel ${ }^{3} S_{1}^{[1]}$ as $p_{T}^{-8}$ at LO, but it is $p_{T}^{-6}$ at next-to-leading order (NLO) and $p_{T}^{-4}$ at next-to-next-to-leading order (NNLO). Thus high order contributions will be dominant when $\alpha_{s} \frac{p_{T}^{2}}{m_{O}^{2}} \gg 1$ for ${ }^{3} S_{1}^{[1]}$ channel. Similarly, ${ }^{1} S_{0}$ and ${ }^{3} P_{J}$ channels behave as $p_{T}^{-6}$ at LO and $p_{T}^{-4}$ at NLO, so NLO corrections for these channels are also very important. Note that, $p_{T}^{-4}$ is already the leading $p_{T}$ behavior, so higher order corrections for ${ }^{3} S_{1}^{[8]}$ channel should be no longer important. Considering the $p_{T}$ behavior discussed here, it is clear that both LO 
CSM and LO NRQCD may not work well. As a result, when higher order contributions are taken into consideration, CSM or NRQCD can give a reasonable description for quarkonium production at high $p_{T}{ }^{3}$.

\subsection{Color-Singlet Model at higher order}

NLO corrections for yield in ${ }^{3} S_{1}^{[1]}$ channel were first done in 2007 by Campbell, Maltoni, and Tramontano [33]. As expected in the last section, NLO contributions can be larger than LO by orders of magnitude in high $p_{T}$ region (Fig. [3). The polarization of ${ }^{3} S_{1}^{[1]}$ channel at NLO was then calculated by Gong and Wang in 2008 [B4], and the authors found the polarization of ${ }^{3} S_{1}^{[1]}$ channel to change from being transverse at LO to longitudinal at NLO (Fig. (4). The polarization of ${ }^{3} S_{1}^{[1]}$ channel at NLO can be understood in collinear factorization in [ए]], where it was shown that the longitudinal contributions have a large logarithm $\log \left(p_{T}^{2} / m_{Q}^{2}\right)$ enhancement compared to transverse contributions. Although the CS NLO contributions can provide large enhancement for $J / \psi$ production at high $p_{T}$, the CSM predictions are still much lower than experimental data.
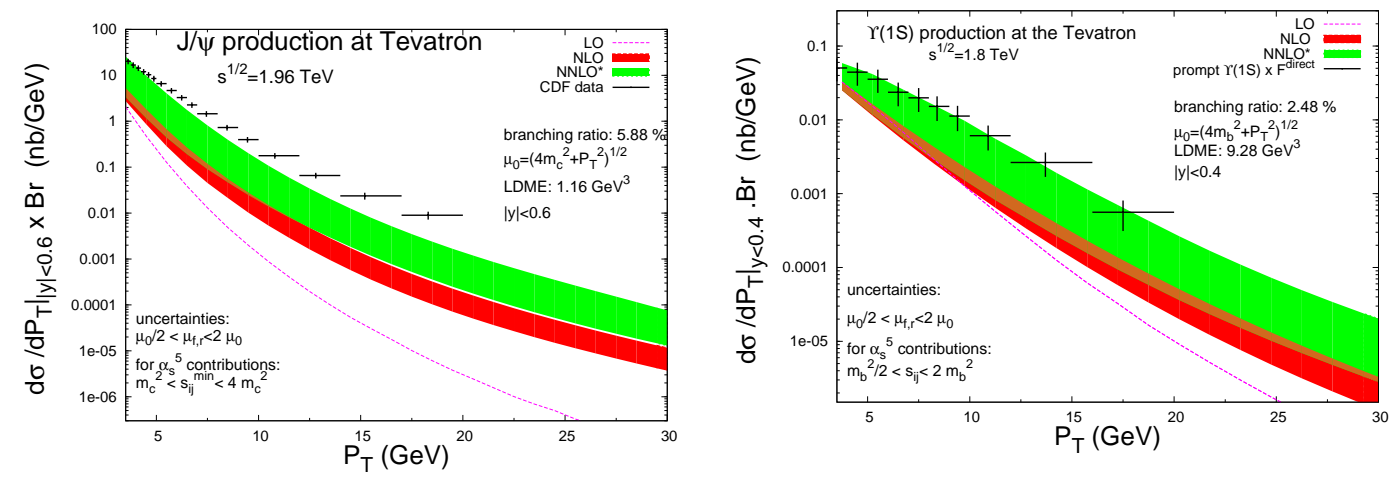

Figure 3: Calculations of the $J / \psi(\Upsilon(1 S))$ production cross sections in the color-singlet channel compared with CDF Run II data [B2] ([B5]). LO: leading order in $\alpha_{s}$. NLO: next-to-leading-order in $\alpha_{s}$. NNLO*: next-to-next-to-leading order in $\alpha_{s}$ but only with real corrections. (Figures taken from Ref.[B6], based on the results by P. Artoisenet, J. Campbell, J.P. Lansberg, F. Maltoni, and F. Tramontano [37].)

Encouraged by the large enhancement at NLO, Artoisenet, Campbell, Lansberg, Maltoni, and Tramontano further calculated the NNLO contributions [B], [88]. Because the complete NNLO calculation for the ${ }^{3} S_{1}^{[1]}$ channel is currently beyond the state of the art, instead, the NNLO ${ }^{\star}$ method is employed in Ref. [B], [B8], where only tree level Feynman diagrams at NNLO are taken into account. An infrared cutoff $s_{i j}^{\min }$ at $O\left(m_{Q}^{2}\right)$ is introduced to control the soft and collinear divergencies, that is, the invariant masses of all pairs larger than $s_{i j}^{\min }$ are required. The NNLO ${ }^{\star}$ contributions are then found to be very large compared with NLO contributions (Fig. [3]), and the Tevatron data for $\Upsilon(1 S)$ can almost be described [B]].

However, Ma, Wang, and Chao in Refs. [39, 40], found that the $\mathrm{NNLO}^{\star}$ result seems to be dominated by contributions that are proportional to $\log ^{2}\left(p_{T}^{2} / s_{i j}^{\min }\right)$ when $p_{T}^{2} \gg s_{i j}^{\min }$. These double

\footnotetext{
${ }^{3}$ The better way to deal with these $p_{T}$ enhancement at higher order is to reorganize the expansion sequence, that is,

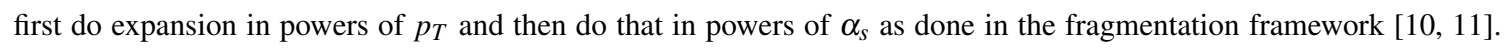
However, predictions using this method are still missing.
} 

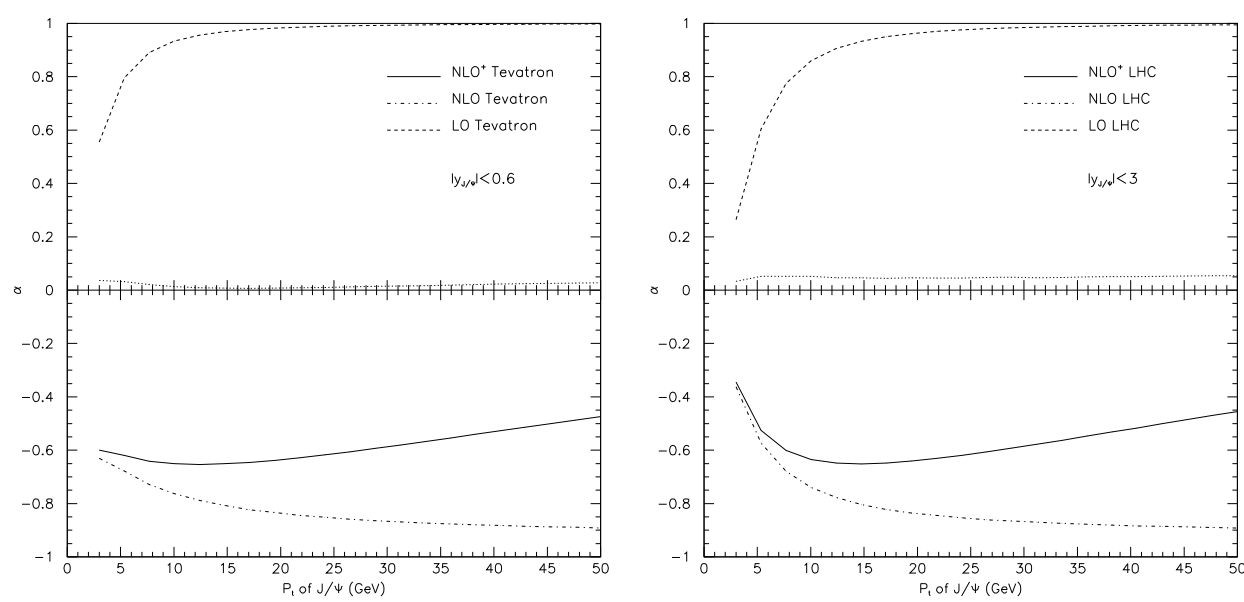

Figure 4: Transverse momentum distribution of differential cross section with $\mu_{r}=\mu_{f}=\sqrt{\left(2 m_{c}\right)^{2}+p_{t}^{2}}$ at LHC (upper curves) and Tevatron (lower curves). Central mass energies are $\sqrt{s}_{\text {Tevatron }}=1.98 \mathrm{TeV}$ and $\sqrt{s}_{\mathrm{LHC}}=14 \mathrm{TeV}$. $\mathrm{NLO}^{+}$denotes result including contribution from $g g \rightarrow J / \psi c \bar{c}$ at NLO. (Figures taken from Ref. [B4].)

logarithm enhanced terms will be cancelled when virtual corrections are included in the complete NNLO calculation. Therefore, complete NNLO result might not have large enhancement compared with NLO, thus CSM cannot explain the observed production cross section of S-wave quarknioum states at high $p_{T}$. Furthermore, as NNLO ${ }^{3} S_{1}^{[1]}$ channel may not be important, complete NLO calculations in NRQCD including both color-singlet and color-octet channels should be done to give predictions for quarkonium production, which will be shown in the following sections.

\subsection{NRQCD factorization at NLO: yield of $J / \psi$}

The complete NLO calculation for $J / \psi$ production up to $O\left(\alpha_{s} v^{4}\right)$ within NRQCD factorization is done by two groups at the same time: Ma, Wang, and Chao [B9] and Butenschöen and Kniehl [43], while NLO corrections for only S-wave channels were done earlier by Gong, Li, and Wang [45]. The NRQCD short distance coefficients of Ma, Wang, Chao and Butenschöen, Kniehl agree. However, the procedures of fitting NRQCD LDMEs are different between the two groups:

- Procedure of Ma, Wang, and Chao: select only data that can be very safely described by perturbation theory to fit LDMEs. Using CDF RunII data with $p_{T}>7 \mathrm{GeV}$ and subtracting feeddown contributions from $\psi^{\prime}$ and $\chi_{c J}$ [42], Ma, Wang, and Chao found only two linear combinations of LDMEs can be well constrained:

$$
\begin{aligned}
& M_{0, r_{0}}^{J / \psi}=\left\langle\mathscr{O}^{J / \psi}\left({ }^{1} S_{0}^{[8]}\right)\right\rangle+\frac{r_{0}}{m_{c}^{2}}\left\langle\mathscr{O}^{J / \psi}\left({ }^{3} P_{0}^{[8]}\right)\right\rangle=(7.4 \pm 1.9) \times 10^{-2} \mathrm{GeV}^{3}, \\
& M_{1, r_{1}}^{J / \psi}=\left\langle\mathscr{O}^{J / \psi}\left({ }^{3} S_{1}^{[8]}\right)\right\rangle+\frac{r_{1}}{m_{c}^{2}}\left\langle\mathscr{O}^{J / \psi}\left({ }^{3} P_{0}^{[8]}\right)\right\rangle=(0.05 \pm 0.03) \times 10^{-2} \mathrm{GeV}^{3},
\end{aligned}
$$

where $r_{0}=3.9$ and $r_{1}=-0.56$ were determined by decomposing the CO P-wave channel for CDF case [39, 40]]. 

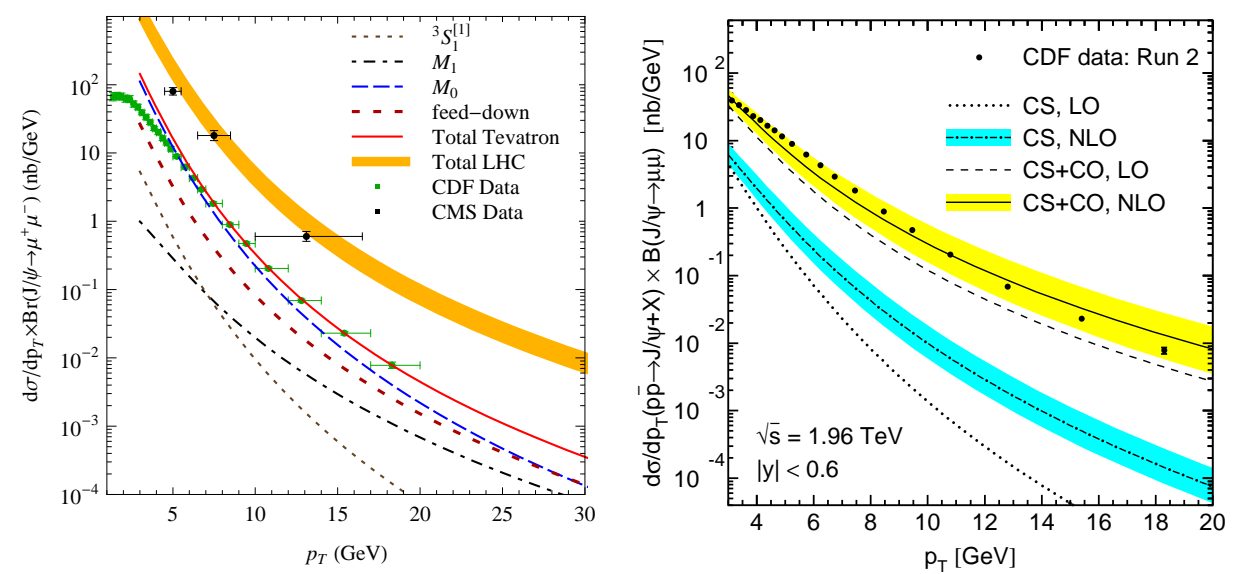

Figure 5: Comparison of NLO NRQCD fits with CDF prompt $J / \psi$ data [4]]]. Left: The fit of Ma, Wang, and Chao [42, [39, 40], feed down contributions are subtracted. (Figure taken from Ref. [39]].) Right: The global fit of Butenschöen and Kniehl, feed down contributions are not subtracted [43], 44]]. (Figure taken from Ref. [43].)

- Procedure of Butenschöen and Kniehl: fit as many as possible data to determine CO LDMEs. Using prompt $J / \psi$ production data in $p p\left(p_{T}>3 \mathrm{GeV}\right)$, ep $\left(p_{T}>1 \mathrm{GeV}\right), \gamma \gamma$, and $e^{+} e^{-}$ collisions, Butenschöen and Kniehl did a global fit to determine all three LDMEs:

$$
\begin{aligned}
\left\langle\mathscr{O}^{J / \psi}\left({ }^{1} S_{0}^{[8]}\right)\right\rangle & =(4.76 \pm 0.06) \times 10^{-2} \mathrm{GeV}^{3}, \\
\left\langle\mathscr{O}^{J / \psi}\left({ }^{3} S_{1}^{[8]}\right)\right\rangle & =(0.265 \pm 0.014) \times 10^{-2} \mathrm{GeV}^{3}, \\
\left\langle\mathscr{O}^{J / \psi}\left({ }^{3} P_{0}^{[8]}\right)\right\rangle / m_{c}^{2} & =(-0.716 \pm 0.089) \times 10^{-2} \mathrm{GeV}^{3},
\end{aligned}
$$

which gives

$$
\begin{aligned}
& M_{0, r_{0}}^{J / \psi}=(2.17 \pm 0.56) \times 10^{-2} \mathrm{GeV}^{3}, \\
& M_{1, r_{1}}^{J / \psi}=(0.62 \pm 0.08) \times 10^{-2} \mathrm{GeV}^{3},
\end{aligned}
$$

which are significantly different from the results in Eq.(3.]). Note that in [43] feed down contributions are not subtracted when using the direct production prediction to fit the prompt production data.

Differences of the fits between these two groups have been discussed in detail in Refs. [39, 40, 43], 46].

Comparison of the NLO NRQCD fits with CDF prompt $J / \psi$ data [4] ] are shown in Fig. [1, where one finds that the Ma, Wang, and Chao fit can well describe data of $p_{T}>7 \mathrm{GeV}$ while the Butenschöen and Kniehl fit can describe all data of $p_{T}>3 \mathrm{GeV}$ within errors. However, the shape of the Ma, Wang, and Chao fit agrees with the data better than that of the Butenschöen and Kniehl fit. This can be more clearly seen in Fig. 6 when predictions confront with even higher $p_{T}$ data . 

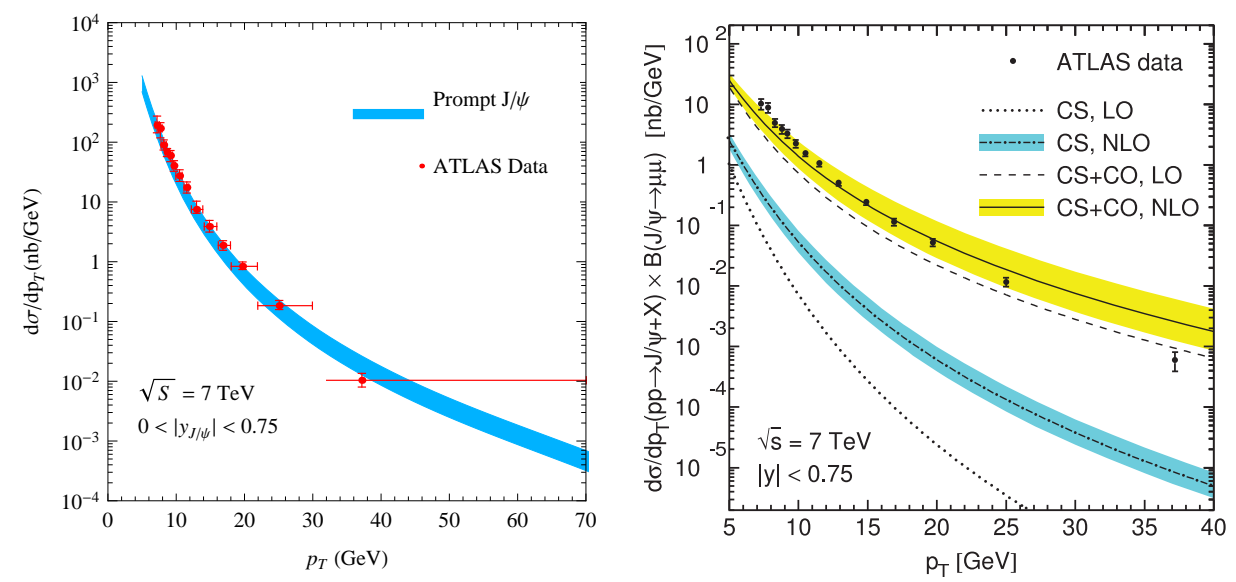

Figure 6: Comparison of NLO NRQCD predictions with the ATLAS prompt $J / \psi$ data [47]]. Left: The prediction of Ma, Wang, and Chao [42], [39, 40]], feed down contributions are subtracted. (Figure taken from Ref. [BQ].) Right: The prediction using global fit of Butenschöen and Kniehl, feed down contributions are not subtracted [43, [4]]. (Figure taken from Ref. [36].)

\subsection{NRQCD factorization at NLO: polarization of $J / \psi$}

The first complete NLO calculations of $J / \psi$ polarization were carried out by two groups at the same time: Butenschöen and Kniehl [48] and Chao, Ma, Shao, Wang, and Zhang [49]. Using their global fit, Butenschöen and Kniehl found the prediction for polarization of $J / \psi$ at the Tevtatron Run-II disagrees with data, which is shown on the left of Fig. W. Chao et.al., however, found there are still parameters space to reconcile the yield and polarization of $J / \psi$ production at the Tevatron, as shown on the right of Fig. $\square$. Note that both of the two groups did not consider feeddown contributions for polarization.
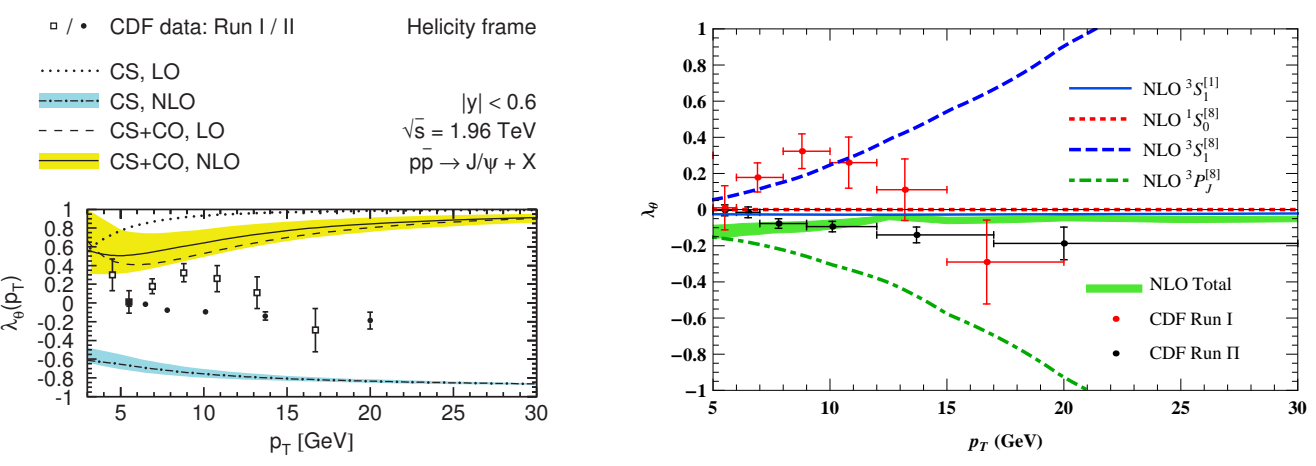

Figure 7: Comparison of NLO NRQCD fits to the CDF polarization of prompt $J / \psi$ data [B], B2]. Left: The fit of Butenschöen and Kniehl [48]], feed down contributions are not subtracted. (Figure taken from Ref. [48].) Right: The fit of Chao, Ma, Shao, Wang, and Zhang, feed down contributions are not subtracted [49]. (Figure taken from Ref. [49].)

Recently, Gong, Wan, Wang, and Zhang considered also the feeddown contributions [50]. 
They found that their results are consistent with CDF Run-I data (except two points), but in conflict with the CDF Run-II data, as shown in Fig. 8. Note that in [50] polarization of prompt $J / \psi$ and polarization of direct $J / \psi$ seem to be almost the same in Fig. 8 . Therefore, the difference of predictions by three groups may come from the different treatments of polarization of direct $J / \psi$. That is, each group gets a different set of LDMEs of $J / \psi$ by fitting data.

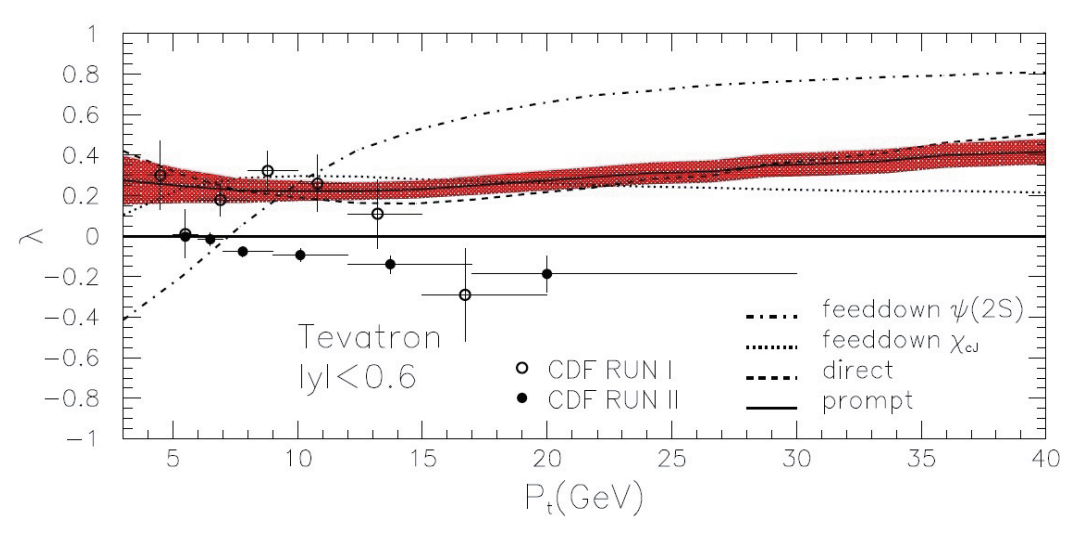

Figure 8: Comparison of NLO NRQCD prediction by Gong, Wan, Wang, and Zhang with the CDF polarization of prompt $J / \psi[30$, B2] $]$. Feeddown contributions are subtracted. (Figure taken from Ref. [50].)

As Gong et.al. used the same fit method developed by Ma, Wang, and Chao in Refs. [42, B9, [40], it is easy to compare the difference of LDMEs by these two groups. By fitting both CDF and LHCb yield of prompt $J / \psi\left(\right.$ with $p_{T}>7 \mathrm{GeV}$ ), Gong et.al. got [5]]

$$
\begin{aligned}
\left\langle\mathscr{O}^{J / \psi}\left({ }^{1} S_{0}^{[8]}\right)\right\rangle & =(9.7 \pm 0.9) \times 10^{-2} \mathrm{GeV}^{3}, \\
\left\langle\mathscr{O}^{J / \psi}\left({ }^{3} S_{1}^{[8]}\right)\right\rangle & =(-0.46 \pm 0.13) \times 10^{-2} \mathrm{GeV}^{3}, \\
\left\langle\mathscr{O}^{J / \psi}\left({ }^{3} P_{0}^{[8]}\right)\right\rangle / m_{c}^{2} & =(-0.95 \pm 0.25) \times 10^{-2} \mathrm{GeV}^{3},
\end{aligned}
$$

which leads to

$$
\begin{aligned}
& M_{0, r_{0}}^{J / \psi}=(6.0 \pm 1.0) \times 10^{-2} \mathrm{GeV}^{3}, \\
& M_{1, r_{1}}^{J / \psi}=(0.07 \pm 0.01) \times 10^{-2} \mathrm{GeV}^{3},
\end{aligned}
$$

which are in good agreement with the fit by Ma, Wang, and Chao in Eq. (B.D. This means that the two well constrained linear combinations of LDMEs are consistent with each other for the two groups.

It is also needed to point out that Chao et.al. imposed a constraint that each LDME must be positive, which reduces the uncertainty and, in particular, gives a prediction that is roughly consistent with the CDF Run-I and Run-II polarization data, namely the $J / \psi$ is approximately unpolarized. This is the consequence of cancellation between ${ }^{3} S_{1}^{[8]}$ and ${ }^{3} P_{J}^{[8]}$ for the transverse polarization contributions [49]. While in works of Butenschöen and Kniehl [48] and Gong et.al. [50], the value of $\left\langle\mathscr{O}^{J / \psi}\left({ }^{3} P_{0}^{[8]}\right)\right\rangle$ is negative. 


\subsection{NRQCD factorization at NLO: $\chi_{c J}, \psi^{\prime}$ and $\Upsilon$}

For $\chi_{c J}$ production, the LO NRQCD prediction is dominated by CO channel at high $p_{T}$, and gives $R_{\chi_{c}} \equiv \sigma\left(\chi_{c 2}\right) / \sigma\left(\chi_{c 1}\right) \rightarrow 5 / 3$ when $p_{T} \gg m_{c}$. However, measurement by CDF shows a very different behavior [5] (Fig. Q). As expected in Sec. B.], NLO corrections are found to be also very important at high $p_{T}$ for this issue. By including NLO contributions, Ma, Wang, and Chao can fit the CDF data by choosing CO LDMEs to satisfy $r=\frac{\left\langle\mathscr{O}^{x_{c 0}(}\left(S_{1}^{[8]}\right)\right\rangle}{\left\langle\mathscr{O}_{c 0}\left(3_{0} P_{0}^{1 !}\right)\right\rangle}=0.27 \pm 0.06$ [42]. Using

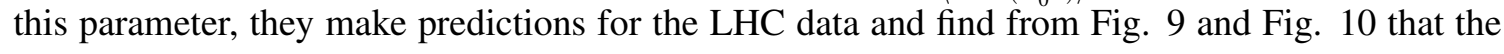
predictions for $\sigma\left(\chi_{c 2}\right) / \sigma\left(\chi_{c 1}\right)$ at CMS [52] and LHCb [53] and $\sigma\left(\chi_{c J}\right) / \sigma(J / \psi)$ at $\mathrm{LHCb}$ [54] are in good agreement with data.
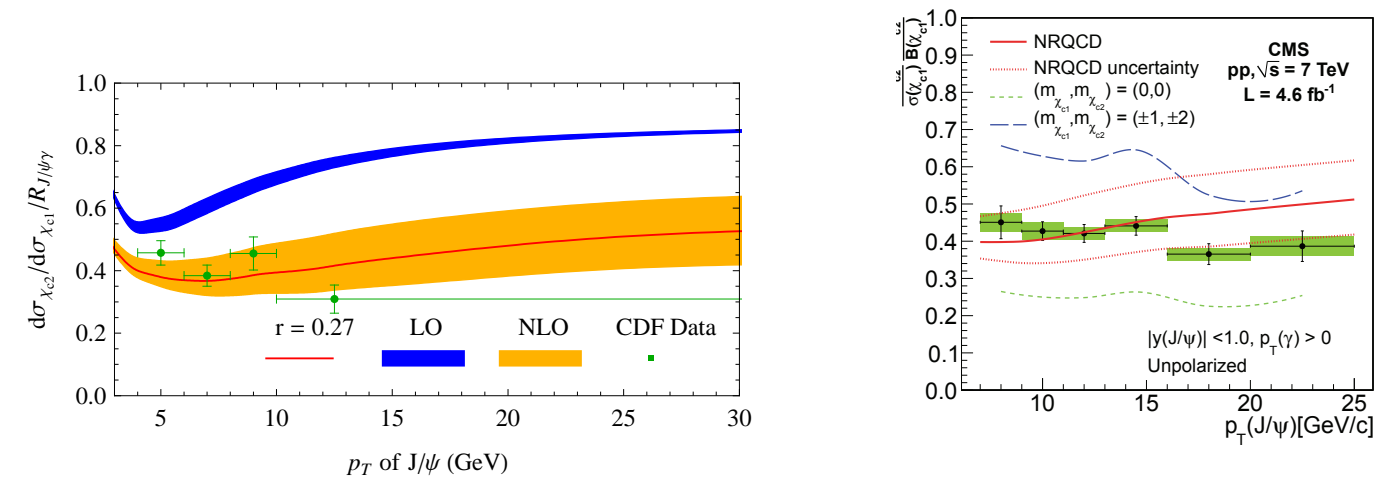

Figure 9: Transverse momentum distribution of ratio $R_{\chi_{c}}$ at the Tevatron and LHC with cut $\left|y_{J / \psi}\right|<1$. The CDF data are taken from Ref.[5]], and CMS data from Ref. [52]. The lower and upper bounds of LO and NLO NRQCD predictions are constrained by $0.24<r<0.33$. (The left figure is from Ref. [42], and the right figure from Ref. [52].)
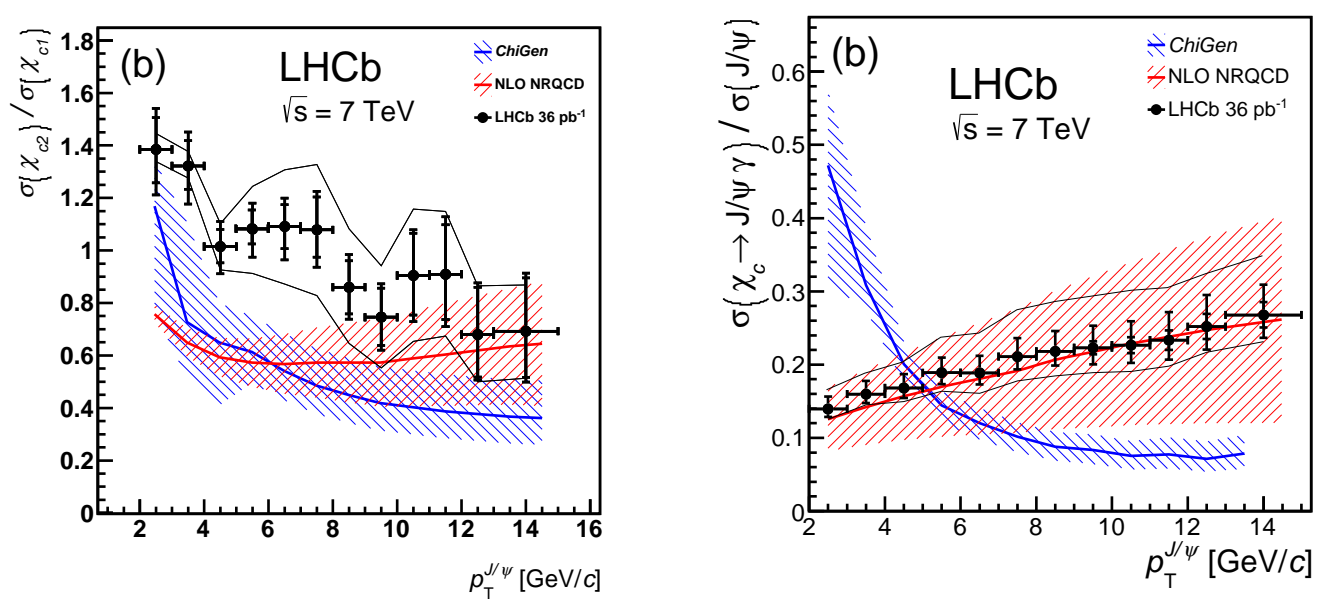

Figure 10: Comparison of predictions for the transverse momentum distributions of ratio $\sigma\left(\chi_{c J}\right) / \sigma(J / \psi)$ and ratio $\sigma\left(\chi_{c 2}\right) / \sigma\left(\chi_{c 1}\right)$ by NLO NRQCD [42] with the LHCb data. (The left figure is from Ref. [53], and the right figure from Ref. [54].) 
The first complete NLO NRQCD calculation for the prompt $\psi^{\prime}(\Upsilon(1 S))$ production is done in Refs. [39, 40] (Ref. [55]). All data for yields of $\psi^{\prime}$ and $\Upsilon(1 S)$ at RHIC, Tevatron, and LHC can be consistently described by NLO NRQCD. As the shapes are similar to that of $J / \psi$, we do not plot diagrams here, but refer interesting readers to Refs. [40, [5]] for details.

\section{Quarkonium production in $e p$ and $\gamma \gamma$ collisions}

For $J / \psi$ photoproduction, the HERA data are roughly consistent with the global fit by Butenschöen and Kniehl [43], 44] for both yield and polarization, only with a slight difference in shape. As shown in Fig. ㅁ], the data are overshot by the the fit of Ma, Wang, and Chao [39, 40]].
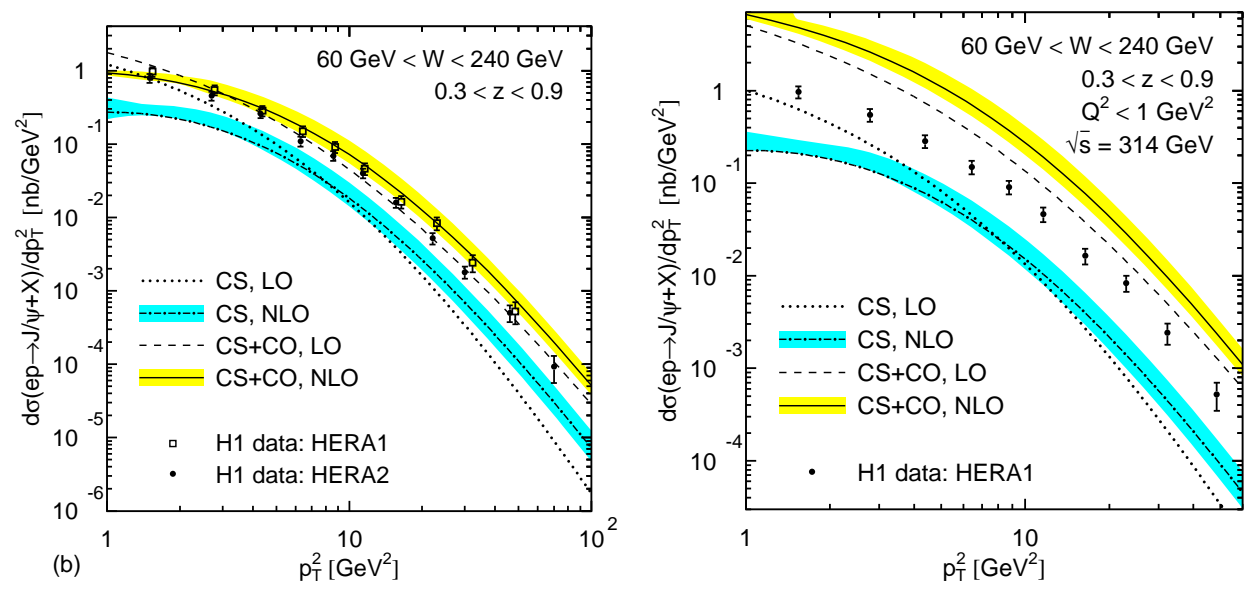

Figure 11: Comparison of the global fit of Butenschöen and Kniehl [43], 44] (left) and the fit by Ma, Wang, and Chao [39, 50] (right) with the H1 data [56, 57])(The left figure is from Ref. [44]], and the right figure from Ref. [B]].)

Butenschöen and Kniehl have also given an updated prediction for $J / \psi$ production in $\gamma \gamma$ collisions at LEPII using their new LDMEs obtained by the global fit [4]]. As shown on the left of Fig. 12, where the DELPHI data are incompatible with this global fit. However, as shown on the right of Fig. [2], the DELPHI data are compatible with an older sets of LDMEs extracted in Ref. [29], where $M_{0,3.4}^{J / \psi}=6.6 \sim 8.7 \times 10^{-2} \mathrm{GeV}^{3}$ is close to the value extracted by Ma, Wang, and Chao in Eq. (B. (ل). Hence, the LEP data favors the value of $M_{0, r_{0}}^{J / \psi}$ from the fit of Ma, Wang, and Chao [B9, 40], rather than the value from the global fit of Butenschöen and Kniehl [43], 44]].

\section{Quarkonium production in $e^{+} e^{-}$annihilation at B factories}

Charmonium production in $e^{+} e^{-}$annihilation at $\mathrm{B}$ factories has provided interesting tests for NRQCD. For exclusive double charmonium production such as $e^{+} e^{-} \rightarrow J / \psi \eta_{c}$ the puzzle of observed unexpectedly large rate [60, 6]], compared with LO calculations [62, 63, 64, 65]], has been largely resolved by NLO corrections in $\alpha_{s}$ [6] and relativistic corrections in $v^{2}$ [67, 68]. The exclusive production strongly indicates the importance of NLO corrections in CS channels but is irrelevant to $\mathrm{CO}$ channels. On the other hand, inclusive charmonium production in $e^{+} e^{-}$annihilation can test both CS and CO mechanisms. While $e^{+} e^{-} \rightarrow J / \psi c \bar{c}$ is dominated by CSM and can be 

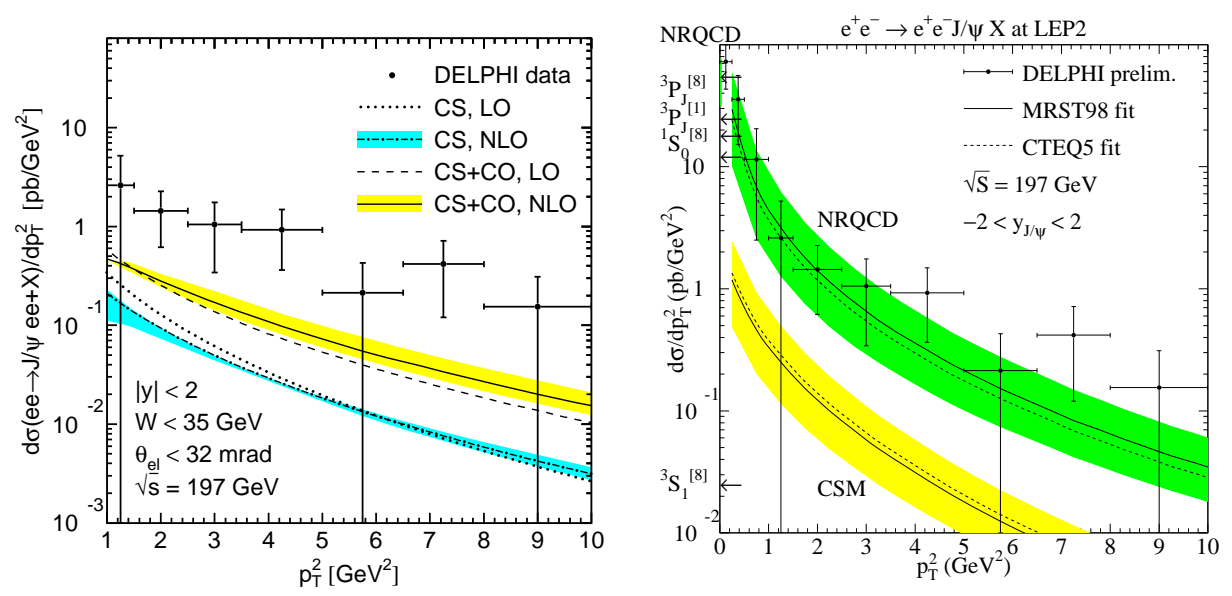

Figure 12: Comparison of the global fit of Butenschöen and Kniehl [4]] (left) and an older prediction by Klasen, Kniehl, Mihaila, and Steinhauser [ [58] using the fit by Braaten, Kniehl and Lee [स] (right) with the DELPHI LEPII data [5]](The left figure is from Ref. [44]], and the right figure from Ref. [58].)

well described by NLO CSM calculations[69], the $J / \psi$ production associated with (non $c \bar{c}$ ) light hadrons can be classified into a CS process $e^{+} e^{-} \rightarrow J / \psi g g$ and a CO process $e^{+} e^{-} \rightarrow(c \bar{c})^{[8]} g$ where $(c \bar{c})^{[8]}$ are $\mathrm{CO}$ intermediate states ${ }^{1} S_{0}^{[8]}$ and ${ }^{3} P_{J}^{[8]}$ which subsequently hadronize to $J / \psi$ by emitting soft gluons. For $e^{+} e^{-} \rightarrow J / \psi g g$ the NLO calculations in $\alpha_{s}$ [四, 孟] as well as in $v^{2}$ [प2] are consistent with the most recent Belle measurement of the $e^{+} e^{-} \rightarrow J / \psi+$ light hadrons cross section [D3], thus leave little room for the $\mathrm{CO}$ contributions from $e^{+} e^{-} \rightarrow(c \bar{c})^{[8]} g$, which then imposes a quite strict constraint on the CO LDMLs of ${ }^{1} S_{0}^{[8]}$ and ${ }^{3} P_{J}^{[8]}$ [सU]]. As a result, both the $M_{0, r_{0}}^{J / \psi}$ given by Ma, Wang, and Chao in Eq. (B.]) and that given by Butenschöen and Kniehl in Eq. (B.3]) are too large to be compatible with the Belle measurement [[23]. However, we note that the Belle observed cross section is smaller by more than a factor of 2 than the earlier BaBar measurement [स5]. Since this experimental discrepancy is significant for constraining the $\mathrm{CO}$ contribution, it should be further clarified.

\section{Summary}

At present, complete NLO predictions in NRQCD for almost all heavy quarkonia (e.g. $J / \psi$, $\chi_{c J}, \psi^{\prime}$, and $\Upsilon$ ) production in high energy collisions are available. The NLO contributions are essential, not just bring about finite $\alpha_{s}$ corrections, but can change the high $p_{T}$ behavior, leading to enhancement by orders of magnitude in hadroproduction. Thus in many cases the theory can give a qualitatively correct description for quarkonium production only after NLO contributions are taken into account. Moreover, the NLO P-wave contributions are very significant. E.g., puzzles about the $\sigma\left(\chi_{c 2}\right) / \sigma\left(\chi_{c 1}\right)$ ratio and the $J / \psi$ polarization might only be resolved when the contributions of P-wave channels at NLO in $\alpha_{s}$ are considered.

However, despite of the success achieved by NRQCD calculations, there seems to be a troublesome problem that the universality of NRQCD LDMEs is in question. It is interesting to note that the data for $J / \psi$ production in $p p$ and $\gamma \gamma$ collisions can be well described by NLO NRQCD with 
a large $M_{0, r_{0}}^{J / \psi}$; whereas the ep and $e^{+} e^{-}$data favor a smaller $M_{0, r_{0}}^{J / \psi}$. Possible ways to understand or to solve the universality problem include:

- Proof of the validity of NRQCD factorization either at some finite order or to all orders is needed.

- Resummation of large logarithms of $\log \left(m_{Q}^{2} / p_{T}^{2}\right)$ is needed to improve theoretical predictions in the low $p_{T}$ region. Some preliminary work has been done recently [प], $\left.\square\right]$ ].

- Resummation of large logarithms of $\log \left(p_{T}^{2} / m_{Q}^{2}\right)$ is needed to improve theoretical predictions in the high $p_{T}$ region. The method to deal with this problem is given in [ए]], but more specific works are needed.

- High order relativistic corrections are needed, since for charmonium the convergence of $v^{2}$ expansion is not good. For CS channels of $J / \psi$ hadroproduction, $p_{T}^{-4}$ contribution has been calculated to $O\left(v^{4}\right)$ in [8, [9], $p_{T}^{-6}$ to $O\left(v^{2}\right)$ in [प9], $p_{T}^{-8}$ to $O\left(v^{2}\right)$ in [80], and a study of all order in $v^{2}$ is given in [ $[\mathbb{R}]$. All these calculations imply that higher order $v^{2}$ contributions for CS channels are not important. For CO channels $O\left(v^{2}\right)$ corrections have been done in [ $\mathbb{B}^{2}$ ], where $v^{2}$ corrections are found to be significant.

- More accurate and consistent experimental data are needed to clarify theoretical issues.

In the LHC era with copious data available from ATLAS, CMS, ALICE, and LHCb, quarkonium production is expected to be in a new phase for the study of QCD and quarkonium physics.

\section{References}

[1] G. T. Bodwin, E. Braaten, and G. P. Lepage Phys. Rev. D51 (1995) 1125 [Erratum-ibid. D 55 (1997) 5853.

[2] N. Brambilla, S. Eidelman, B. Heltsley, R. Vogt, G. Bodwin, et al. Eur.Phys.J. C71(2011) 1534.

[3] V. Kartvelishvili, A. Likhoded, and S. Slabospitsky Sov.J.Nucl.Phys. 28 (1978) 678.

[4] C.-H. Chang Nucl.Phys. B172 (1980) 425-434.

[5] E. L. Berger and D. L. Jones Phys. Rev. D23 (1981) 1521 .

[6] H. Fritzsch Phys. Lett. B67 (1977) 217.

[7] F. Halzen Phys. Lett. B69 (1977) 105.

[8] J. F. Amundson, O. J. P. Eboli, E. M. Gregores, and F. Halzen Phys. Lett. B372 (1996) 127.

[9] J. F. Amundson, O. J. P. Eboli, E. M. Gregores, and F. Halzen Phys. Lett. B390 (1997) 323 .

[10] Z.-B. Kang, J.-W. Qiu, and G. Sterman Nucl.Phys.Proc.Suppl. 214 (2011) 39-43.

[11] Z.-B. Kang, J.-W. Qiu, and G. Sterman Phys.Rev.Lett. 108 (2012) 102002.

[12] G. T. Bodwin, E. Braaten, and J. Lee Phys. Rev. D72 (2005) 014004.

[13] G. C. Nayak, J.-W. Qiu, and G. F. Sterman Phys. Rev. D72 (2005) 114012.

[14] G. C. Nayak, J.-W. Qiu, and G. F. Sterman Phys. Lett. B613 (2005) 45. 
[15] G. C. Nayak, J.-W. Qiu, and G. F. Sterman Phys. Rev. Lett. 99 (2007)212001.

[16] G. C. Nayak, J.-W. Qiu, and G. F. Sterman Phys. Rev. D77 (2008) 034022.

[17] E. Braaten, K.-m. Cheung, and T. C. Yuan Phys. Rev. D48 (1993) 4230.

[18] E. Braaten and J. Lee Nucl.Phys. B586 (2000) 427-439.

[19] G. T. Bodwin, U.-R. Kim, and J. Lee arXiv:1208.5301 hep-ph].

[20] CDF Collaboration Collaboration arXiv: hep-ex/9412013 hep-ex].

[21] M. Cacciari and M. Greco Phys. Rev. Lett. 73 (1994) 1586.

[22] E. Braaten, M. A. Doncheski, S. Fleming, and M. L. Mangano Phys. Lett. B333 (1994) 548.

[23] D. Roy and K. Sridhar Phys.Lett. B339 (1994) 141-147.

[24] M. Krämer Prog. Part. Nucl. Phys. 47 (2001) 141.

[25] E. Braaten and S. Fleming Phys. Rev. Lett. 74 (1995) 3327.

[26] M. Cacciari, M. Greco, M. L. Mangano, and A. Petrelli Phys.Lett. B356(1995) 553-560.

[27] P. L. Cho and M. B. Wise Phys.Lett. B346 (1995) 129-136.

[28] M. Beneke and I. Rothstein Phys.Lett. B372 157-164 [Erratum-ibid. B 389 (1996) 769].

[29] E. Braaten, B. A. Kniehl, and J. Lee Phys. Rev. D62 (2000) 094005.

[30] CDF Collaboration, A. A. Affolder et al. Phys. Rev. Lett. 85 (2000) 2886.

[31] S. P. Baranov Phys.Rev. D66 (2002) 114003.

[32] CDF Collaboration, A. Abulencia et al. Phys. Rev. Lett. 99 (2007) 132001.

[33] J. M. Campbell, F. Maltoni, and F. Tramontano Phys. Rev. Lett. 98 (2007) 252002.

[34] B. Gong and J.-X. Wang Phys. Rev. Lett. 100 (2008) 232001.

[35] CDF Collaboration, D. E. Acosta et al. Phys. Rev. Lett. 88 (2002) 161802.

[36] G. T. Bodwin arXiv:1208.5506 hep-ph].

[37] P. Artoisenet, J. M. Campbell, J. P. Lansberg, F. Maltoni, and F. Tramontano Phys. Rev. Lett. 101] 2008) 152001.

[38] J. P. Lansberg Eur. Phys. J. C61 (2009) 693.

[39] Y.-Q. Ma, K. Wang, and K.-T. Chao Phys. Rev. Lett. $106(2011) 042002$.

[40] Y.-Q. Ma, K. Wang, and K.-T. Chao Phys.Rev. D84 (2011) 114001.

[41] CDF Collaboration, D. Acosta et al. Phys. Rev. D71 (2005) 032001.

[42] Y.-Q. Ma, K. Wang, and K.-T. Chao Phys. Rev. D83 (2011) III503(R).

[43] M. Butenschoen and B. A. Kniehl Phys. Rev. Lett. $106(2011) 022003$.

[44] M. Butenschoen and B. A. Kniehl Phys.Rev. D84 (2011)051501.

[45] B. Gong, X. Q. Li, and J.-X. Wang Phys. Lett. B673 (2009) 197 [Erratum-ibid. 693, 612 (2010)].

[46] M. Butenschoen and B. A. Kniehl AIP Conf.Proc. 1343 (2011) 409-411.

[47] ATLAS Collaboration Collaboration, G. Aad et al. Nucl.Phys. B850 (2011) 387-444. 
[48] M. Butenschoen and B. A. Kniehl Phys.Rev.Lett. 108 (2012) 172002.

[49] K.-T. Chao, Y.-Q. Ma, H.-S. Shao, K. Wang, and Y.-J. Zhang Phys.Rev.Lett. 108 (2012) 242004.

[50] B. Gong, L.-P. Wan, J.-X. Wang, and H.-F. Zhang arXiv:1205.6682 [hep-ph].

[51] CDF Collaboration, A. Abulencia et al. Phys. Rev. Lett. 98 (2007) 232001.

[52] CMS Collaboration Collaboration, S. Chatrchyan et al. arXiv:1210.0875 hep-ex].

[53] LHCb Collaboration Collaboration, R. Aaij et al. Phys.Lett. B714(2012) 215-223.

[54] LHCb Collaboration Collaboration, R. Aaij et al. Phys.Lett. B718 (2012) 431-440.

[55] K. Wang, Y.-Q. Ma, and K.-T. Chao Phys.Rev. D85 (2012) 114003 .

[56] H1 Collaboration, C. Adloff et al. Eur. Phys. J. C25 (2002) 25.

[57] H1 Collaboration, F. D. Aaron et al. Eur. Phys. J. C68 (2010) 401.

[58] M. Klasen, B. A. Kniehl, L. N. Mihaila, and M. Steinhauser Phys. Rev. Lett. 89 (2002) 032001.

[59] DELPHI Collaboration, J. Abdallah et al. Phys. Lett. B565 (2003) 76.

[60] Belle Collaboration, K. Abe et al. Phys. Rev. Lett. 89(2002) 142001.

[61] BABAR Collaboration, B. Aubert et al. Phys. Rev. D72 (2005) 031101.

[62] E. Braaten and J. Lee Phys. Rev. D67 (2003) 054007.

[63] K.-Y. Liu, Z.-G. He, and K.-T. Chao Phys. Lett. B557 (2003) 45.

[64] K. Hagiwara, E. Kou, and C.-F. Qiao Phys.Lett. B570 (2003) 39-45.

[65] K.-Y. Liu, Z.-G. He, and K.-T. Chao Phys.Rev. D77 (2008) 014002.

[66] Y.-J. Zhang, Y.-J. Gao, and K.-T. Chao Phys. Rev. Lett. 96 (2006) 092001.

[67] G. T. Bodwin, D. Kang, and J. Lee Phys. Rev. D74 (2006) 114028.

[68] Z.-G. He, Y. Fan, and K.-T. Chao Phys. Rev. D75 (2007) 07401].

[69] Y.-J. Zhang and K.-T. Chao Phys. Rev. Lett. $98(2007) 092003$.

[70] Y.-Q. Ma, Y.-J. Zhang, and K.-T. Chao Phys. Rev. Lett. 102 (2009) 162002.

[71] B. Gong and J.-X. Wang Phys. Rev. Lett. 102 (2009) 162003.

[72] Z.-G. He, Y. Fan, and K.-T. Chao Phys.Rev. D81 (2010) 054036.

[73] Belle Collaboration Collaboration, P. Pakhlov et al. Phys.Rev. D79 (2009) 071101].

[74] Y.-J. Zhang, Y.-Q. Ma, K. Wang, and K.-T. Chao Phys. Rev. D81 (2010) 034015.

[75] BABAR Collaboration Collaboration, B. Aubert et al. Phys.Rev.Lett. 87 (2001) 162002.

[76] P. Sun, C.-P. Yuan, and F. Yuan arXiv:1210.3432 Thep-ph].

[77] J. Ma, J. Wang, and S. Zhao arXiv: 1211.7144 hep-ph].

[78] G. T. Bodwin and J. Lee Phys.Rev. D69(2004) 054003.

[79] K.-T. Chao, J.-Z. Li, and Y.-Q. Ma In preparation .

[80] Y. Fan, Y.-Q. Ma, and K.-T. Chao Phys. Rev. D79 (2009) 114009.

[81] Y.-Q. Ma arXiv:1207.3073 hep-ph].

[82] G.-Z. Xu, Y.-J. Li, K.-Y. Liu, and Y.-J. Zhang arXiv:1203.0207 [hep-ph]. 